\title{
Developments in controlled environmental transfer for Li-based battery materials: From sample preparation to SEM investigation
}

\author{
Pawel Nowakowski, Cecile Bonifacio, Mary Ray and Paul Fischione
}

\section{E.A. Fischione Instruments, Inc., United States}

The fundamental components of Li ion batteries (LIBs) are the anode, separator, cathode, and electrolyte. Battery types can be differentiated by the electrolyte used: liquid electrolyte solution versus solid electrolyte, the latter of which also serves as an anode and cathode separator.

LIBs are widely used in electric vehicles and mobile devices; therefore, improving both LIB battery capacity and safety are a significant concern. Regarding safety, commercialized liquid electrolyte LIBs are susceptible to electrolyte solution leakage caused by external force damage. In addition, they pose a risk of explosion or fire due to overcharging. Solid-state electrolyte LIBs (SSE LIBs) promise increased capacity through the use of Li metal anode and safe operation through the use of a non-flammable solidstate electrolyte [1]. However, degradation over the course of the SSE LIB lifecycle remains an issue because the aging process constrains energy storage capabilities and performance. Better understanding of the degradation mechanisms and structural changes during battery lifetime is critical to improving SSE LIB functionality and performance [1-3].

Sample preparation is critical for microstructural investigations of any material. Sample preparation at ambient atmosphere is impossible because SSE LIB components are extremely sensitive to oxygen, nitrogen, water, and carbon dioxide. SSE LIB components are also comprised of soft materials (e.g., Li is low density and ductile; SSE is a compacted powder), the microstructure of which can be easily damaged by various sample preparation techniques.

A broad ion beam milling technique in a controlled environment makes SSE LIB sample preparation possible. Because Li metal and SSE have an additional vulnerability - both are temperature sensitive cryogenic conditions are required during ion milling (Fig. 1).

The characteristics of SSE LIB demand a controlled environmental workflow from sample preparation to SEM analyses $[4,5]$. We report on instrument developments that allow the direct transfer of the sample from an ion milling system [Model 1062 TrionMill, Fischione Instruments] to SEM without use of a glove box. The sample preparation system's transfer mechanism allows transfer at vacuum, in inert gas, or in a cryogenic environment. When transferring a sample at vacuum, the mechanism's active pumping capability maintains vacuum from the ion mill to the SEM. The efficacy of the direct transfer is illustrated by pure lithium metal preparation in the ion milling system and subsequent SEM investigation [Fig. 1b]. The high-quality electron backscatter diffraction patterns (EBSD) [Fig. 2a] and collected large-area EBSD maps confirm neither oxidation nor contamination of the sample during transfer [Fig. 2b]. The methodology was also applied to a more complex Li-based battery system and will be presented. 

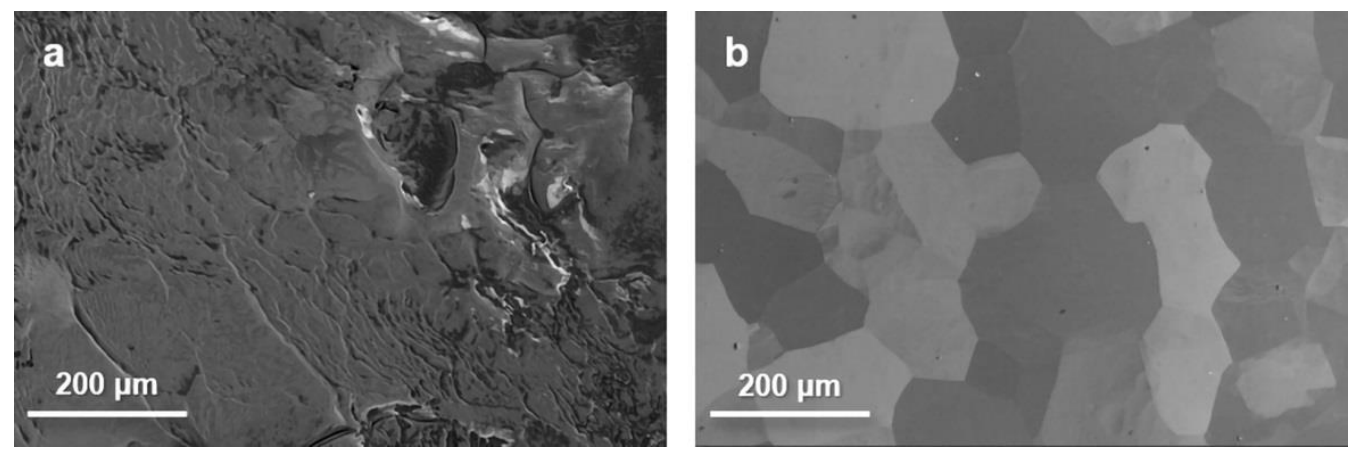

Figure 1. SEM images of a pure Li metal following argon ion milling at $6 \mathrm{keV}$ after transfer to the SEM under Ar atmosphere. Sample showing surface damage following ion milling at ambient temperature (a); sample showing grain microstructure following ion milling at cryogenic temperatures (b).
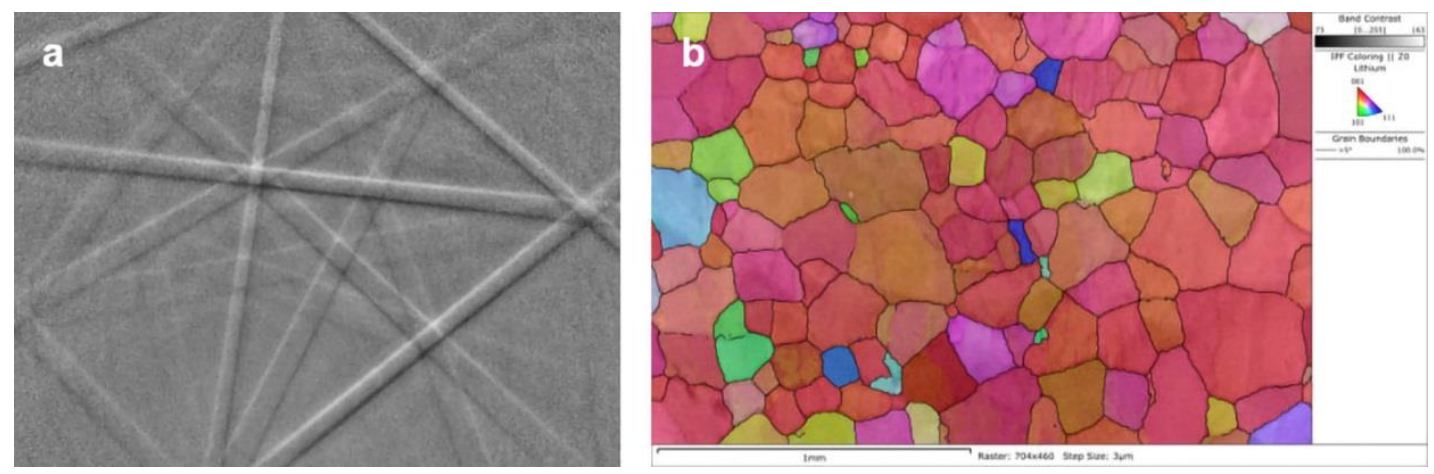

Figure 2. Figure 2. EBSD pattern obtained from Li metal sample after ion milling under cryogenic conditions (a). Inverse pole figure color-coded map with grain boundary network overlay on band contrast EBSD map (b). Map was collected at 20 $\mathrm{kV}$ using $3 \mu \mathrm{m}$ step size.

\section{References}

1. Banerjee, A., Wang, X., Fang, C., Wu, E. A., \& Meng, Y. S. (2020). Interfaces and interphases in allsolid-state batteries with inorganic solid electrolytes. Chemical Reviews, 120(14), 68786933. https://doi.org/10.1021/acs.chemrev.0c00101

2. Nie, K., Hong, Y., Qiu, J., Li, Q., Yu, X., Li, H., \& Chen, L. (2018). Interfaces between cathode and electrolyte in solid state lithium batteries: Challenges and perspectives. Frontiers in Chemistry, 6, Article ID 616, 19 pages. https://doi.org/10.3389/fchem.2018.00616

3. Lin, C., Tang, A., Mu, H., Wang, W., \& Chung, C. (2015). Aging mechanisms of electrode materials in lithium-ion batteries for electric vehicles. Journal of Chemistry, 2015, Article ID 104673, 11 pages. https://doi.org/10.1155/2015/104673

4. Bonifacio, C., Nowakowski, P., Ray, M., \& Fischione, P. (2020). Multi-length scale cryogenic sample preparation to electron microscopy of battery materials. Microscopy and Microanalysis, 26(S2), 30223024. https://doi.org/10.1017/S1431927620023569

5. Nowakowski, P., Bonifacio, C. S., Doux, J., Meng, S., Ray, M. L., \& Fischione, P. (2020, November). Gaining a better understanding of lithium ion battery functionality through scanning electron microscopy analytical studies[Conference session]. Materials Research Society 2020 Fall Meeting, Virtual, in press. 